\title{
COVID-19 vaccine hesitancy in South Africa: A complex social phenomenon
}

The COVID-19 pandemic continues to have significant health, human, social and economic impacts on South African (SA) society. COVID-19 vaccines currently offer the most promising means to safeguard personal health, protect the most vulnerable populations, reopen social and economic life, and potentially achieve population health and safety through immunity. ${ }^{[1]}$ However, the success of COVID-19 vaccines depends on high levels of uptake. Supply-side factors related to vaccine availability, infrastructure and accessibility all pose significant challenges to optimal uptake in SA. ${ }^{[2,3]}$ Vaccine hesitancy - which involves varying levels of doubt, indecision, uncertainty or mistrust about vaccination ${ }^{[4]}$ - is an additional challenge. Vaccine hesitancy poses significant risks, not only for the hesitant individual, but also for the wider community.

With the COVID-19 pandemic and current roll-out of COVID19 vaccines, vaccine hesitancy has gained renewed attention as an important national public health concern in SA. Delays or refusals of COVID-19 vaccination could mean that the country is unable to reach the thresholds of vaccine uptake necessary for herd immunity. ${ }^{[5]}$ Vaccine hesitancy research in the country has also gained considerable momentum, with numerous surveys having been conducted over the past year to explore acceptance of COVID-19 vaccines among South Africans. ${ }^{[6]}$

One of the central findings emerging from these surveys is that uptake of a biomedical intervention such as a vaccine is a complex social phenomenon. COVID-19 vaccination views in SA are influenced by social factors such as age, race, education, politics, geographical location and employment. For example, a number of surveys found that age may be an important factor, with younger adults potentially having more concerns and/or being less accepting of COVID-19 vaccination than those who are older ${ }^{[7,8]}$ (also see research by Ask Afrika, 'COVID-19 Tracker: Unpacking the significant social change brought on by the COVID-19 pandemic', supplementary file at https://www.askafrika.co.za/wp-content/ uploads/2021/05/Ask-Afrika-Covid-Tracker-week-1-Vaccines. pdf, and 'COVID-19 Tracker: A gender report on South Africa', supplementary file at https://www.askafrika.co.za/wp-content/ uploads/2021/05/Ask-Afrika-COVID-19-Omnibus-Week-1-2021GENDER.pdf). Race, ${ }^{[7]}$ education ${ }^{[7]}$ and urbanicity ${ }^{[8,9]}$ were also identified in various surveys as playing a role in shaping COVID-19 vaccine acceptance, with white adults, people with higher levels of education and those living in more rural areas being less accepting of the vaccine than other groups. Views about COVID-19 vaccines were also found to be influenced by political factors. ${ }^{[7,8]}$ More specifically, political discontent or disillusionment was identified as potentially important - people who had positive attitudes towards the government generally and its handling of COVID-19 in particular were more likely to accept COVID-19 vaccination.

This social nature of vaccination views is not unique to COVID-19 - public views about a diverse range of vaccines and vaccination programmes have been found to be an inherently social phenomenon. ${ }^{[10,11]}$ For example, in a recently conducted global Cochrane review of qualitative evidence, we found that views and practices around childhood vaccination reflected multiple webs of influence, meaning and logic - social, political, structural, moral and biological. ${ }^{[12]}$ Our review revealed how, through their vaccination choices, parents are often communicating not just what they think about vaccines, but also who they are, what they value and with whom they identify. Based on these findings, we concluded that vaccination, whatever stance is taken, is a complex social process that is deeply embedded in the wider social worlds in which people live.

Taking these social worlds seriously and placing them at the centre of efforts to reduce hesitancy and promote COVID-19 vaccine acceptance in SA are critical. In particular, such efforts need to involve more than information and education, and factor in that people develop their vaccine beliefs through their life experiences and that culture, structural conditions, personal background, religion and politics all shape people's reactions to facts supplied to them. ${ }^{[6]}$ Properly understanding, and grounding interventions within, localised contexts and value systems are therefore important. Such efforts should also recognise that vaccine beliefs are often rooted in distrust of institutions, and associated historical and contemporary experiences of inequality, injustice and exploitation. Incorporating broader trust-building measures that focus on communication and dialogue, relationships, transparency and community involvement and participation is therefore important. Such efforts also need to be cognisant of the fact that people often have many needs and priorities, and that acceptance of vaccines may depend on these other concerns also being met. Integrating vaccine hesitancy reduction initiatives within broader health and development programmes is therefore important. ${ }^{[13]}$ Most certainly, these kinds of initiatives are unlikely to translate into onedimensional and 'quick-fix' interventions. Yet we believe that they are essential if we hope to respond effectively and sensitively to public ambiguities and uncertainties around COVID-19 vaccines in SA.

\section{S Cooper}

Cochrane South Africa, South African Medical Research Council, Cape Town, South Africa; and School of Public Health and Family Medicine, Faculty of Health Sciences, University of Cape Town, South Africa

sara.cooper@mrc.ac.za

\section{H van Rooyen}

Human Sciences Research Council, Pretoria, South Africa; and MRC-Wits Developmental Pathways for Health Research Unit, Faculty of Health Sciences, University of the Witwatersrand, Johannesburg, South Africa

\section{S Wiysonge}

Cochrane South Africa, South African Medical Research Council, Cape Town, South Africa; School of Public Health and Family Medicine, Faculty of Health Sciences, University of Cape Town, South Africa; and Department of Global Health, Faculty of Medicine and Health Sciences, Stellenbosch University, Cape Town, South Africa

1. National Academies of Sciences, Engineering and Medicine (NASEM). Strategies for building confidence in the COVID-19 vaccines. Washington, DC: National Academies Press, 2021. https://dol. org $/ 10.17226 / 26068$

2. Gray G, Van der Heever A, Madhi SS, et al. The Scientists' Collective 10-point proposal for equitable and timeous access to COVID-19 vaccine in South Africa. S Afr Med J 2021;111(2):89-94. https://doi. org/10.7196/SAMI.2021.v111i2.15498

3. Moodley K, Rossouw T. What could fair allocation of an efficacious COVID-19 vaccine look like in South Africa? Lancet Glob Health 2021;9(2):e106-e107. https://doi.org/10.1016//2214-

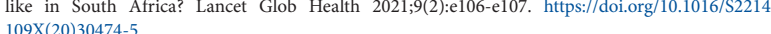

4. World Health Organization. Report of the SAGE Working Group on Vaccine Hesitancy, 1 October 2014 http://www.who.int/immunization/sage/meetings/2014/october/1_Report_WORKING_GROUP vaccine_hesitancy_final.pdf (accessed 1 March 2018) 

https://doi.org/10.1093/cid/cir007

6. National Department of Health, South Africa. Strategies to address COVID-19 vaccine hesitancy and promote acceptance in South Africa. Covid-19 Online Resource and News Portal, 12 Augus 2021. https://sacoronavirus.co.za/2021/04/12/strategies-to-address-covid-19-vaccine-hesitancy-andpromote-acceptance-in-south-africa/ (accessed 27 April 2021)

7. Runciman C, Roberts B, Alexander K, Bohler-Muller N, Bekker M. UJ-HSRC Covid-19 Democracy Survey. Willingness to take a Covid-19 vaccine: A research briefing. University of Johannesburg, Survey. Willingness to take a Covid-19 vaccine: A research briefing. University of Johannesburg,
Centre for Social Change, Human Sciences Research Council, and Developmental, Capable and Ethical Centre for Social Change, Human Sciences Research Council, and Developmental, Capable and Ethical
State, 25 January 2021. https://www.uj.ac.za/newandevents/PublishingImages/Pages/UJ-HSRCState, 25 January 2021. https://www.uj.ac.za/newandevents/PublishingImages/Pages/UJ-HSRC-
survey-shows-that-two-thirds-of-adults-are-willing-to-take-the-Covid-19-vaccine/2021-01-25\%20 survey-shows-that-two-thirds-of-adults-are-willing-to-take-
Vaccine\%20briefing\%20(final).pdf (accessed 2 February 2021)

8. Africa Centres for Disease Control (CDC). COVID-19 vaccine perceptions: A 15-country study 10 March 2021. https://africacdc.org/download/covid-19-vaccine-perceptions-a-15-country-study/ (accessed 15 May 2021)

9. Willie M, Skosana E. COVID-19 vaccines report: Medical schemes member survey. Policy, Research and Monitoring, Council for Medical Schemes, March 2021. https://www.medicalschemes.co.za/pressrelease-3-of-2021-cms-releases-the-covid-19-vaccines-survey-results/ (accessed 2 May 2021).
10. Larson HJ, Jarrett C, Eckersberger E, Smith DM, Paterson P. Understanding vaccine hesitancy around vaccines and vaccination from a global perspective: A systematic review of published literature, 2007 2012. Vaccine 2014;32(19):2150-2159. https://doi.org/10.1016/j.vaccine.2014.01.081

11. Dube E, Gagnon D, MacDonald N, Bocquier A, Peretti-Watel P, Verger P. Underlying factors impacting vaccine hesitancy in high income countries: A review of qualitative studies. Expert Rev Vaccines 2018;17(11):989-1004. https://doi.org/10.1080/14760584.2018.1541406

12. Cooper S, Schmidt BM, Sambala EZ, et al. Factors that influence parents' and informal caregivers' acceptance of routine childhood vaccination: A qualitative evidence synthesis. Cochrane Database Syst Rev 2019, Issue 2. Art. No.: CD013265. https://doi.org/10.1002/14651858.CD013265

13. Harrison EA, Wu JW. Vaccine confidence in the time of COVID-19. Eur J Epidemiol 2020;35(4):325330. https://doi.org/10.1007/s10654-020-00634-3

S Afr Med J 2021;111(8):702-703. https://doi.org/10.7196/SAMJ.2021.v111i8.15800 